\title{
Nodes With the Highest Control Power Play an Important Role at the Final Level of Cooperation in Directed Networks
}

\author{
Ali Ebrahimi \\ University of Tehran \\ Marzieh Yousefi \\ Isfahan University of Technology \\ Farhad Shahbazi \\ Isfahan University of Technology \\ Ali Masoudi-Nejad ( $\nabla$ amasoudin@ut.ac.ir) \\ University of Tehran
}

\section{Research Article}

Keywords: Controllability, network, control centrality, public goods game, level of cooperation

Posted Date: March 1st, 2021

DOI: https://doi.org/10.21203/rs.3.rs-263967/v1

License: (c) (i) This work is licensed under a Creative Commons Attribution 4.0 International License. Read Full License 


\title{
Nodes with the highest control power play an important role at the final level of cooperation in directed networks
}

\author{
Ali Ebrahimi ${ }^{1}$, Marzieh Yousefi ${ }^{2}$, Farhad Shahbazi $^{2}$, Ali Masoudi-Nejad ${ }^{{ }^{*}}$ \\ 1. Laboratory of Systems Biology and Bioinformatics (LBB), Institute of Biochemistry and \\ Biophysics, University of Tehran, Tehran, Iran \\ 2. Department of physics, Isfahan University of Technology (IUT), Isfahan, Iran
}

*Corresponding Author

Ali Masoudi-Nejad, Ph.D,

Laboratory of Systems Biology and Bioinformatics (LBB)

Institute of Biochemistry and Biophysics

University of Tehran, Tehran, Iran.

E-mail: amasoudin@ut.ac.ir

WWW: http://LBB.ut.ac.ir 


\begin{abstract}
Controllability of complex networks seeks the lowest number of nodes (driver nodes) that controlling all nodes by receiving input signals. The concept of control centrality is used to determine the power of each nodes in the control of the network. The more nodes a node controls through connections in the network, the more the power to control. In the public goods game, cooperative and free-rider strategies are considered and the final level of cooperation in a population is studied. However, it is yet to determine a solution for indicating the effectiveness of each member to change other member's strategies. In a network, the choice of nodes effective in changing other nodes strategy, as free-riders, will lead to lower cooperation and vice versa. This paper uses simulated and real networks to investigate the effectiveness of nodes with the highest control power in changing other nodes strategies and the final level of cooperation compared to the hubs, local, and random nodes. Results indicate that choosing the nodes with the highest control power as free-riders will lead to a lower level of cooperation and is, therefore, more effective in changing the strategies of other nodes, compared to other sets in consideration. This result can be considered in the treatment of cancer; with the explanation that, destroying tumoral cells with the highest control power should be a priority as these cells have a higher capability in changing the strategy of other cells from cooperators to free-riders (healthy to tumoral).
\end{abstract}

Keywords: Controllability, network, control centrality, public goods game, level of cooperation 


\section{Introduction}

In modeling the complex systems by networks, the system agents are considered as nodes and the relationships between them as edges. Network analysis is possible in two ways: static and dynamic. In a static analysis, the time-independent topologic properties of a network are calculated. These topologic properties can be classified into two groups: global and individual. Examples of global topologic properties include distribution of node degrees, path length, degree correlation, network diameter. These properties specify how the entire system behaves and acts as a whole. Individual topologic features indicate each network element's importance as nodes, edges, motifs, and modules. Examples of these properties are power and centrality measures, closeness, betweenness, bridging, and control centrality, and each of them indicates the difference in specificity and the importance of nodes in maintaining network stability.

The dynamic analysis focuses on the network components' temporal evolution in contrast to the static analysis methods. What is essential in analyzing the dynamic of networks is the collective behavior of agents living in them ${ }^{1}$. There are various theoretical methods for improving synchronous movement performance in dynamic networks. Changing the node relationship structure in the network, increasing the weight of links between the nodes, adding or removing edges to or from the network are the examples of the most common methods ${ }^{2}$. However, most of the methods mentioned above cannot be implemented in a network due to their limitations. An efficient method for improving the synchronous movement's performance on the networks is the external control of the nodes.

A dynamical system is called controllable when the proper choice of inputs can transfer the system from any initial state to the desired one through a finite number of $\operatorname{steps}^{3-5}$. The main problem in controllability is finding the least number of nodes in the networks that enable controlling all nodes when affected by input signals. Targeted controllability searches for the least number of nodes in the network that can control a particular target.

To find the driver nodes in directed networks, the minimum input theorem based on Lin's structural controllability theorem ${ }^{6}$ indicates the relationship between structural controllability and maximum matching ${ }^{7}$. Moreover, in order for target control, a greedy algorithm has been provided based on multiple maximal matching ${ }^{8}$. Target controllability is an NP-hard problem ${ }^{9}$, 
and many algorithms have been developed to improve its outcomes ${ }^{10-15}$. In target controllability, placement of non-target nodes in control signals' pathway leads to side effects in many applications. Minimizing the intermediate nodes between driver nodes and target nodes is among the most demanding controllability problems. To this end, we use an algorithm which is based on the path length between each pair of nodes in a directed network. In this algorithm, the highest number of target nodes controlled by each node is given by considering the least intermediates between the driver and target nodes ${ }^{14}$.

Control centrality is an individual topologic characteristic in a network used to determine each node's power in the control of the network ${ }^{16}$. In other words, the more a node can control other nodes via connections in the network, the higher it ranks in terms of control centrality and vice versa. The importance of control and the applications of finding the driver nodes have been subject to much attention ${ }^{17-23}$.

Public goods game is a group game inspecting the collaboration among members of a population using competition among different groups. A colleague (cooperator) and a free-rider (defector) are two existing strategies randomly distributed in the population ${ }^{24}$. Collaborators (C) pay a cost for collaboration, which the free-riders (D) do not, like paying a tax in a society where some pay the tax, but everyone reaps the benefits.

In this manuscript, we show that the nodes with the highest control power have a critical role in the outcome of a public goods game, such that if selected as free-riders, they more strongly direct the population towards betrayal.

\section{Methods}

Consider a directed network $\mathrm{G}(\mathrm{A})$ with $\mathrm{N}$ nodes, whose dynamics is given by the following linear differential equation:

$$
d x(t) / d(t)=A x(t)+B u(t)
$$


In which $A_{N \times N}$ is the connectivity matrix between the nodes. The $x(t)=\left(x_{1}(t), \ldots, x_{N}(t)\right)^{T}$ vector determines the state of $N$ nodes at the moment $t . B_{N \times M}(M \leq N)$ indicates the driver nodes, where in its columns, the elements corresponding to the driver node are nonzero while all other elements are zero. The network is controlled via a time-dependent incoming vector $u(t)=$ $\left(u_{1}(t), \ldots, u_{M}(t)\right)^{5,25}$.

The main problem in controllability is finding a proper subset of network nodes with the lowest cardinality, such that when they undergo input signals, they take all network nodes to the desired state. These nodes are called driver nodes.

Based on the Kalman condition ${ }^{3}$, the network is controlled via nodes related to the B matrix, if $Q_{N \times N M}=\left[B|A B| A^{2} B|\ldots| A^{N-1} B\right]$ has a complete rank, i.e., $\operatorname{rank}(Q)=N$.

The controllability method was used to determine the number of nodes under the control of each node. The method is based on the path lengths between each node pair. In this method, for every node in the network, the maximum number of nodes under control is achieved if the number of the intermediate nodes is minimized ${ }^{14}$. The nodes with the highest control power are then detected and considered as the set of nodes that initially employ a free-rider strategy. To assess nodes' performance with the highest control power, we compare them with the three other groups of nodes with the free-rider strategy:

(1) Nodes with the highest out-degree,

(2) Local selection, for which a node is randomly selected, and then its first, second and ... k-th neighbors, hierarchically, are chosen to be the free-riders.

(3) Random selection of nodes in which every node has an equal probability to be selected as a free-rider.

To start the public goods game, one selects a member of the population as the first player and then considers a group of nodes with a specific size, centered around that member, as its coplayers.

In the public goods game with linear benefit function, the payoff of a defector in the groups is given by: 


$$
P_{D}=\frac{r^{*} j^{*} c}{n}
$$

and for the payoff of a cooperator, we have:

$$
P_{c}=P_{D}-c
$$

In which $r$ is the synergic factor, $j$ is the number of cooperators in a group, $c$ is the cost paid for the beginning of cooperation and $n$ is the group size. In the simplest variant, $n$ is the number of first neighbors plus the player itself. In the next step of the game, we randomly select one of the first player's nearest neighbors and calculate its benefit (the second player). Finally, according to the benefits calculated and considering the selective update rule, decisions will be made about changing the first player's strategy.

The equations (2) and (3) are the linear payoffs commonly used in the standard public goods game. However, nonlinear benefit functions seem to be superior in reality, like biology. In biological processes, such as synthesizing a specific enzyme, secretion stops when a certain enzyme concentration threshold is achieved. It means that enzyme Secretion depends on enzyme concentration in the medium. Generally, most biological processes have a threshold and a saturation limit; hence the benefit function corresponding to these processes, to a large extent, is close to a sigmoid function. It is shown that using a nonlinear benefit function gives rise completely different outcomes than the linear function ${ }^{26}$.

In this work, for the benefit function, we use a sigmoid function given by:

$$
b(j)=\frac{l(j)-l(0)}{l(n)-l(0)}
$$

In which:

$$
l(j)=\frac{1}{1+\exp (s(h-j / n))}
$$


In Eqs. (4) and (5), $j$ is the number of cooperators in a group and is determined by the diffusion length $d^{27}$. $h$ and $s$ are the turning point, and the slope of the payoff function, respectively, and $n$ is the group size.

The benefit functions for a cooperator a defector, in a group containing $\mathrm{j}$ cooperators are given by:

$$
P_{D}=b(j) \& P_{C}=P_{D}-c
$$

We use both the deterministic and the stochastic update rules for updating the first player's strategy. In the deterministic update rule, the first player selects its neighboring co-player strategy with probability 1 if its neighbor gets more payoff. Otherwise, it retains its own strategy. However, in the stochastic (Glauber) update rule, we use the following probability for the switching of the first player strategy:

$$
p(x \rightarrow y)=\frac{1}{1+\exp \left(\beta\left(P_{x}-P_{y}\right)\right.}
$$

where $P_{x}$ and $P_{y}$ are the first and second players' benefits respectively, and $\beta$ controls the level of uncertainty (large $\beta$ corresponds to low noise and vice versa).

\section{Result}

\section{Synthesized Networks}

First, we investigate the public goods game in the directed scale-free networks and also a toy network. The Barabasi-Albert (BA) preferential attachment algorithm ${ }^{28}$ is employed to generate directed scale-free networks with 400 nodes in two structures: sparse $(\langle\mathrm{k}=10\rangle)$ and dense $(<\mathrm{k}=20>)$. Then 20 percent of network nodes (80 nodes) are selected as the initial free-riders. Four groups of nodes are chosen as the initial defectors: 1) nodes with the highest control power, 2) nodes with the maximum out-degree, 3) local nodes, and 4) randomly selected nodes. 
Considering the networks' directional structure, each player can affect only the neighbors' strategy for which there is a directed connection toward them.

Fig 1. represents a toy network, where a directed network with 7 nodes and 9 edges is considered. For each of the aforementioned four condition sets, first two nodes have the D strategy and the affection mechanism on other nodes and their strategy switching is presented step by step. As visible in the picture, selecting the two nodes with the highest control power in the network as free-riders, the network is moved towards complete betrayal and zero cooperation.

Fig 2. parts $b$ and d display the results related to the dynamic behavior of dense and sparse network for deterministic updating. These figures show that the edge density of the network has a remarkable effect on cooperation. Indeed, the level of cooperation is in average $20 \%$ higher in the sparse network.

An important parameter in the public goods game is the cooperation cost (c). Increasing the cost will lead to reluctance in the people to cooperate, and finally, tend to vanish the cooperation at some point. However, in the outcomes yielded, there are cost intervals, for which the level of cooperation shows a plateau. This effect can be explained with regard to network directionality, as in a directed network, one node is affected by the other, only if one directed link exists from the second node to the first.

Fig 2. parts a and c display the final cooperation results yielded from a dense and sparse network. The comparison drawn between these two networks shows that the denser a network is, the less cooperative it becomes. Indeed, controlling the network towards betrayal is easier if the network is denser. The above conclusions are all based on Monte-Carlo calculations, averaged out of 20 independent configurations.

Fig 3. displays the effect of the Glauber update rule on the level of cooperation in the network type. In a dense network, the effect of node predominance with the highest control power is easy to observe for the Glauber random update. Up to a $c=0.6$, for the other three choices of the freerider nodes, the results are the same. For $c>0.6$, however, the effectiveness of random choice can well be observed. Despite this, in the case of selecting hub and local nodes as initial targets of free-rider nodes, results will not vary. As a general outcome, we can say that when we have a 
dense network, regardless of the update rule, there is a higher chance of betrayal than a sparse network.

\section{Real networks}

In this section, we perform the public goods game in the real networks. We use the three goldstandard directed networks: s420 with 252 nodes and 399 edges $^{29}$, s208 with 122 nodes and 189 edges $^{29}$, and Mangrove with 97 nodes and 1492 edges $^{30}$. Results are displayed in Fig 4., Fig S1. and Fig S2. respectively.

The outcomes presented in Fig 4. demonstrate that in a 5420 network in deterministic conditions, transitions occur, similar to what we found in the synthesized networks. Furthermore, in deterministic conditions, the significance of the nodes with the highest control power in changing the strategies of other nodes is clear only for $c>1$. However, In the case of the Glauber update, the role of nodes with the highest control power can be well observed for all values of the cost. For the network dynamics, we can see that the effect of hubs is considerable to reach the steadystate sooner

The results of simulation in the $s 208$ network, presented in Fig S1. indicate that for the deterministic state, the choice of nodes with the highest control power on the network as the initial defectors have a moderate effect on the propagation of betrayal in the network for the intermediate values of cost. This is true, despite the fact that for the Glauber update, these nodes will drive the network towards stabilizing the D strategy, better than other nodes. Fig S1.b and S1.d show that for both deterministic and Glauber updating rules, reaching a steady-state is longer when the nodes with higher control power are selected as the initial defectors.

Fig S2. represents the outcomes of the simulations with the two update rules in the dense Mangrove network. The results shown in this figure indicate that for both updating rules, the selection of all four groups as the initial defectors does no lead to a clear distinction in the final level of cooperation for all values of cost. 


\section{Conclusions}

Cooperation in society is critical in its development and enrichment. The way that the members of a population can cooperate or betray to each-other can affect the outcome of society-related processes. In such conditions, what matters is the strategy change among members of the society who participate in this process. The effort to change one's mind and to be placed among the freeriders deserves attention. For example, in a poll, changing the voter's strategies leads to a change in the election's outcome.

In this paper, we implemented the concept of controllability in the public goods game to control the level of cooperation in a population residing ln a network. The results obtained in both synthesized and real networks indicate that initiating the defector strategy from the set of nodes with higher control power is most effective in changing the strategies of other nodes from cooperation to betrayal.

As an application in cancer treatment, the healthy and tumoral cells can be considered as cooperators and free-riders. The signaling between any two cells forms a link between them from which the complex network corresponding to the inter-cellular communication is defined. The topology of this assembled web determines the control power of each node. Hence, destroying tumoral cells with the highest control power should be a priority as these cells have a higher capability in changing the strategy of other cells from cooperators to free-riders (healthy to tumoral).

Moreover, the presented results demonstrate that the outcomes of the two Glauber and deterministic update rules are different, such that the network shows more resistance against

achieving the D steady state when the Glauber rule is employed. Sparsity or density can also play a role in determining the amount of cooperation. Regardless of the mimicking rule, in a denser network, conditions are more suitable for propagating the D strategy in the network. Therefore, using the deterministic updating and the dense networks exhibit the highest inclination to the $\mathrm{D}$ strategy. 


\section{References}

1 Arenas, A., Díaz-Guilera, A. \& Pérez-Vicente, C. J. Synchronization processes in complex networks. Physica D: Nonlinear Phenomena 224, 27-34 (2006).

2 Jalili, M. Enhancing synchronizability of diffusively coupled dynamical networks: a survey. IEEE transactions on neural networks and learning systems 24, 1009-1022 (2013).

3 Kalman, R. E. Mathematical description of linear dynamical systems. Journal of the Society for Industrial and Applied Mathematics, Series A: Control 1, 152-192 (1963).

4 Luenberger, D. G. Introduction to dynamic systems; theory, models, and applications. (1979).

5 Slotine, J.-J. E. \& Li, W. Applied nonlinear control. Vol. 199 (Prentice hall Englewood Cliffs, NJ, 1991).

6 Lin, C.-T. Structural controllability. IEEE Transactions on Automatic Control 19, 201208 (1974).

7 Liu, Y.-Y., Slotine, J.-J. \& Barabási, A.-L. Controllability of complex networks. nature 473, 167-173 (2011).

8 Gao, J., Liu, Y.-Y., D'souza, R. M. \& Barabási, A.-L. Target control of complex networks. Nature communications 5, 1-8 (2014).

9 Czeizler, E., Gratie, C., Chiu, W. K., Kanhaiya, K. \& Petre, I. in International Conference on Computational Methods in Systems Biology. 67-81 (Springer).

10 Gao, L., Zhao, G., Li, G., Guo, F. \& Zeng, F. Optimal Target Control of Complex Networks with Selectable Inputs. IEEE Transactions on Control of Network Systems (2020).

11 Lu, F., Yang, K. \& Qian, Y. Target control based on edge dynamics in complex networks. Scientific reports 10, 1-12 (2020).

$12 \mathrm{Li}$, G. et al. Target control and expandable target control of complex networks. Journal of the Franklin Institute 357, 3541-3564 (2020).

13 LI, C. \& YAO, H. MOMENT APPROACH TO THE TARGET CONTROL PROBLEM FOR LINEAR SYSTEM. Journal of applied mathematics \& informatics 38, 239-247 (2020).

14 Ebrahimi, A., Nowzari-Dalini, A., Jalili, M. \& Masoudi-Nejad, A. Target controllability with minimal mediators in complex biological networks. Genomics 112, 4938-4944 (2020).

15 Vosughi, A., Johnson, C., Xue, M., Roy, S. \& Warnick, S. Target control and source estimation metrics for dynamical networks. Automatica 100, 412-416 (2019).

16 Perc, M. et al. Statistical physics of human cooperation. Physics Reports 687, 1-51 (2017).

17 Liu, Y.-Y., Slotine, J.-J. \& Barabási, A.-L. Control centrality and hierarchical structure in complex networks. Plos one 7, e44459 (2012).

18 Pósfai, M., Gao, J., Cornelius, S. P., Barabási, A.-L. \& D'Souza, R. M. Controllability of multiplex, multi-time-scale networks. Physical Review E 94, 032316 (2016).

19 Ishitsuka, M., Akutsu, T. \& Nacher, J. C. Critical controllability analysis of directed biological networks using efficient graph reduction. Scientific reports 7, 1-10 (2017).

20 Nacher, J. C. \& Akutsu, T. in Computational Biology of Non-Coding RNA 289-300 (Springer, 2019). 
21 Yan, G. et al. Network control principles predict neuron function in the Caenorhabditis elegans connectome. Nature 550, 519-523 (2017).

22 Asgari, Y., Salehzadeh-Yazdi, A., Schreiber, F. \& Masoudi-Nejad, A. Controllability in cancer metabolic networks according to drug targets as driver nodes. PloS one 8, e79397 (2013).

23 Vinayagam, A. et al. Controllability analysis of the directed human protein interaction network identifies disease genes and drug targets. Proceedings of the National Academy of Sciences 113, 4976-4981 (2016).

24 Meshkati, F. \& Fu, H. C. Modeling rigid magnetically rotated microswimmers: Rotation axes, bistability, and controllability. Physical Review E 90, 063006 (2014).

25 Kailath, T. Linear systems. Vol. 156 (Prentice-Hall Englewood Cliffs, NJ, 1980).

26 Archetti, M. Evolutionary dynamics of the Warburg effect: glycolysis as a collective action problem among cancer cells. Journal of theoretical biology 341, 1-8 (2014).

27 Archetti, M. Dynamics of growth factor production in monolayers of cancer cells and evolution of resistance to anticancer therapies. Evolutionary applications 6, 1146-1159 (2013).

28 Barabási, A.-L. \& Albert, R. Emergence of scaling in random networks. science 286, 509-512 (1999).

29 Milo, R. et al. Network motifs: simple building blocks of complex networks. Science 298, 824-827 (2002).

30 Ulanowicz, R. E. \& DeAngelis, D. L. Network analysis of trophic dynamics in south florida ecosystems. US Geological Survey Program on the South Florida Ecosystem, 114 (1999).

\section{Authors' contribution}

Ali Ebrahimi: Designed the study, implemented, analyzed, and interpreted results, wrote the manuscript. Marzieh Yousefi: Implemented, analyzed, and interpreted results, wrote the manuscript. Farhad Shahbazi: Assisted in interpretations, and revising the manuscript. Ali Masoudi-Nejad: Supervision, assisted in interpretations, and revising the manuscript. All authors approved the final version of the manuscript.

\section{Additional information}

\section{Competing interests:}

The authors declare no competing interests. 


\section{Figure Legends}

Fig 1: The effect of initial choice of free-rider nodes on strategy switching by other network nodes. a) A directed network with 7 nodes and 9 edges. b) Network adjacency matrix. c) H: Nodes with the maximum out-degree, R: Random selection of initial target nodes, L: local selection of nodes, D: Nodes with the highest control power in the network. d) Selection of hub nodes as initial target for the free-rider nodes and analyzing the change in the strategy of other nodes, where the final level of cooperation amounts to $3 / 7$. (In each stage, the free-rider nodes are displayed in red, their immediate neighbors are displayed in yellow and their cooperating nodes are displayed in green). e) The final level of cooperation through random selection of freerider nodes amounts to $2 / 7$. f) By selecting local nodes as free-rider, the final level of cooperation is $3 / 7$. g) Selection of the nodes with the highest control power as free-riders has caused the whole nodes of the network to change their strategy hierarchically and finally the network reaches complete betrayal.

Fig 2: Investigation the effect of initial choice of free-rider nodes in controlling the scalefree networks through deterministic update, for networks with 400 nodes, in dense and sparse networks. a) Analysis of cooperation level in a dense network: Increase in the cooperation cost will lead to decrease in the amount of cooperation. Nodes with the highest control ability, in similar conditions, lead to a lower level of cooperation. b) Analysis of dense network dynamics: if the driver nodes are selected as free-riders, cooperation will decrease $(\mathrm{c}=1)$. c) Analysis of cooperation level in a sparse network: the random sets with the highest control power, whereas by selecting hub and local nodes, a higher level of cooperation is achieved. d) Analyzing the dynamics of a sparse network: just like a dense network, the velocity of achieving a steady state is higher, where nodes with the highest control power are selected $(\mathrm{c}=1)$. 
Fig 3: Analyzing the effect of initial node selection in network control using the Glauber update rule. Other conditions are as the ones shown in Fig 2.

Fig 4: Results regarding to the $\mathbf{5 4 2 0}$ network a) Analysis of cooperation level based on the deterministic update rule. b) Network dynamics in achieving steady state relevant to the deterministic update law ( $c=1)$. c) Analysis of cooperation level based on the Glauber update rule. d) Network dynamics in achieving steady state related to the Glauber update rule (c=1).

Figure 1

a

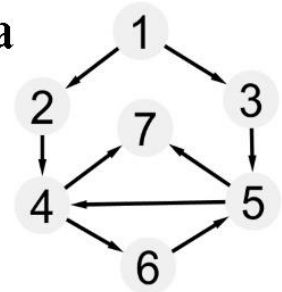

b

$$
A=\left[\begin{array}{lllllll}
0 & 1 & 1 & 0 & 0 & 0 & 0 \\
0 & 0 & 0 & 1 & 0 & 0 & 0 \\
0 & 0 & 0 & 0 & 1 & 0 & 0 \\
0 & 0 & 0 & 0 & 0 & 1 & 1 \\
0 & 0 & 0 & 1 & 0 & 0 & 1 \\
0 & 0 & 0 & 0 & 1 & 0 & 0 \\
0 & 0 & 0 & 0 & 0 & 0 & 0
\end{array}\right]
$$

c
$\mathrm{H}=\{4,5\}$
$\mathrm{R}=\{3,6\}$
$\mathrm{L}=\{5,7\}$
$\mathrm{D}=\{1,2\}$
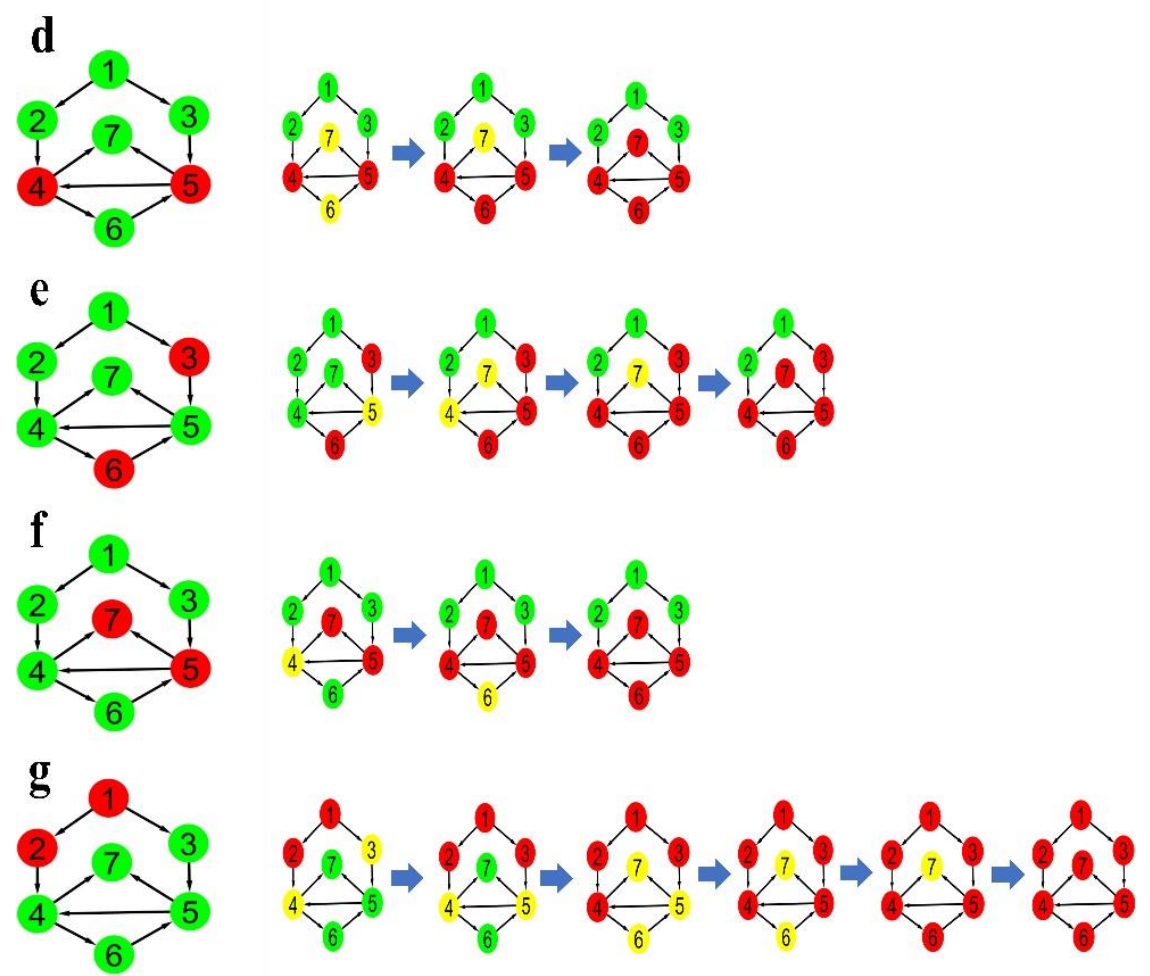
Figure 2

(a)

(c)
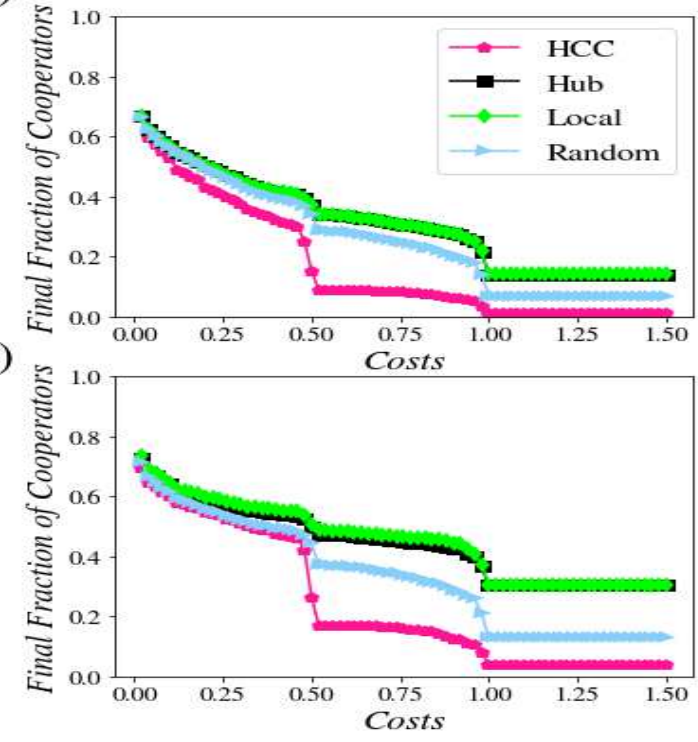

(b)

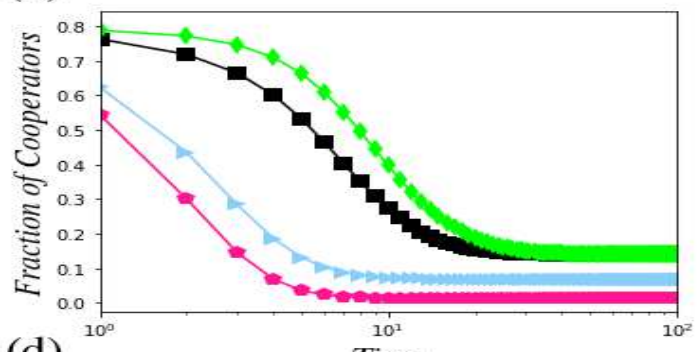

(d)

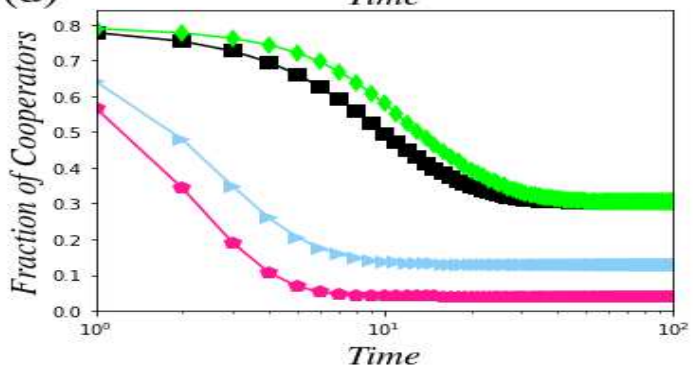

\section{Figure 3}

(a)

(c)

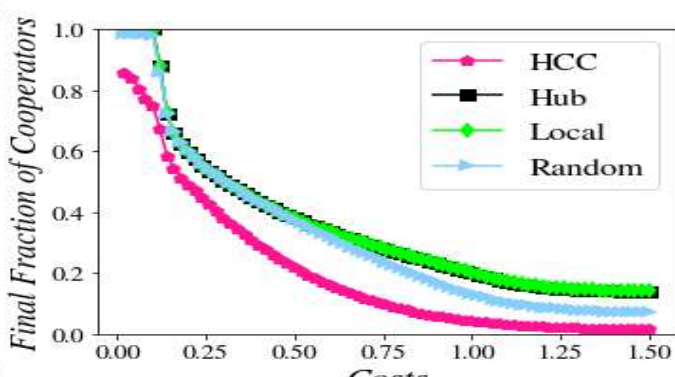

(b)

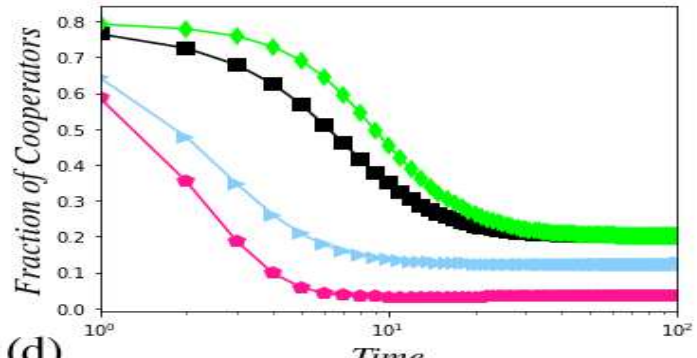

(d)

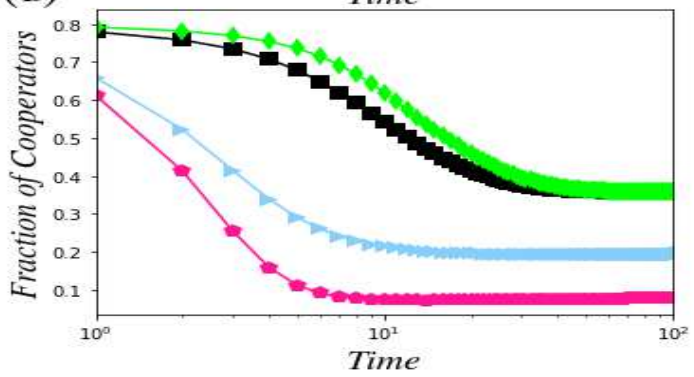




\section{Figure 4}

(a)

(c)
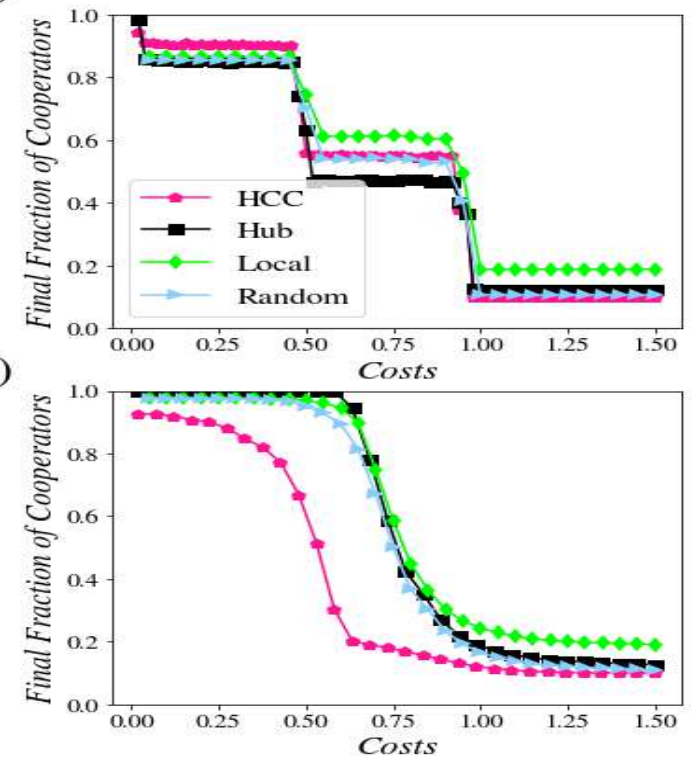

(b)

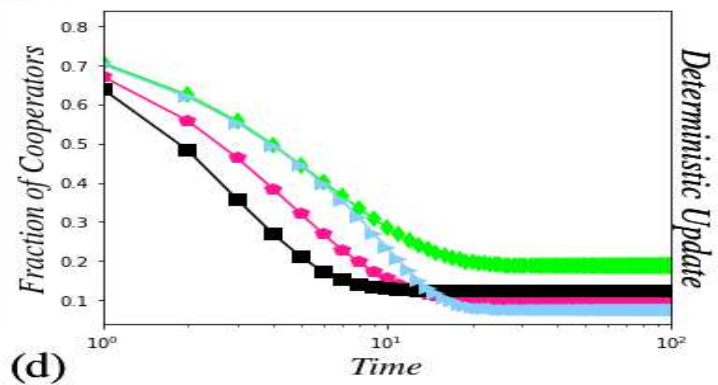

(d)

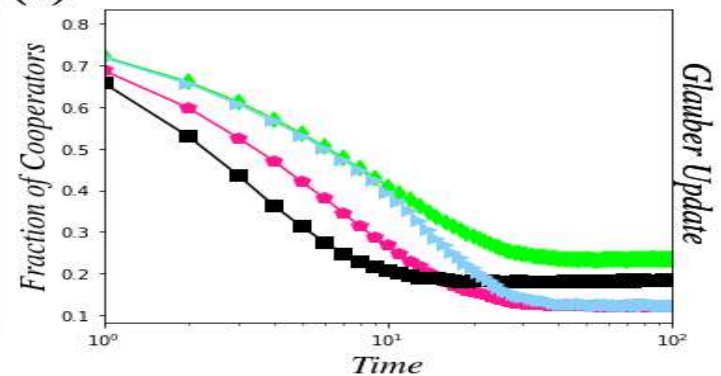


Figures

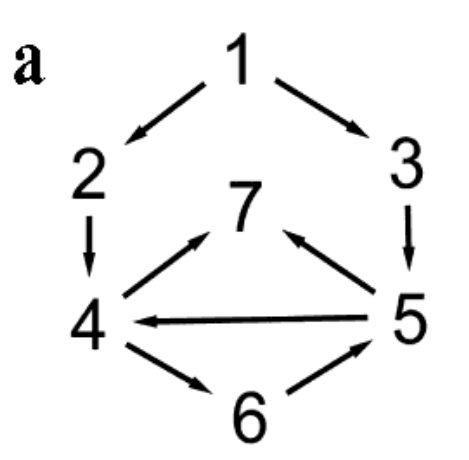

$$
\mathbf{b}=\left[\begin{array}{lllllll}
0 & 1 & 1 & 0 & 0 & 0 & 0 \\
0 & 0 & 0 & 1 & 0 & 0 & 0 \\
0 & 0 & 0 & 0 & 1 & 0 & 0 \\
0 & 0 & 0 & 0 & 0 & 1 & 1 \\
0 & 0 & 0 & 1 & 0 & 0 & 1 \\
0 & 0 & 0 & 0 & 1 & 0 & 0 \\
0 & 0 & 0 & 0 & 0 & 0 & 0
\end{array}\right]
$$

\begin{tabular}{|l|}
\hline $\mathrm{H}=\{4,5\}$ \\
\hline $\mathrm{R}=\{3,6\}$ \\
\hline $\mathrm{L}=\{5,7\}$ \\
\hline $\mathrm{D}=\{1,2\}$ \\
\hline
\end{tabular}
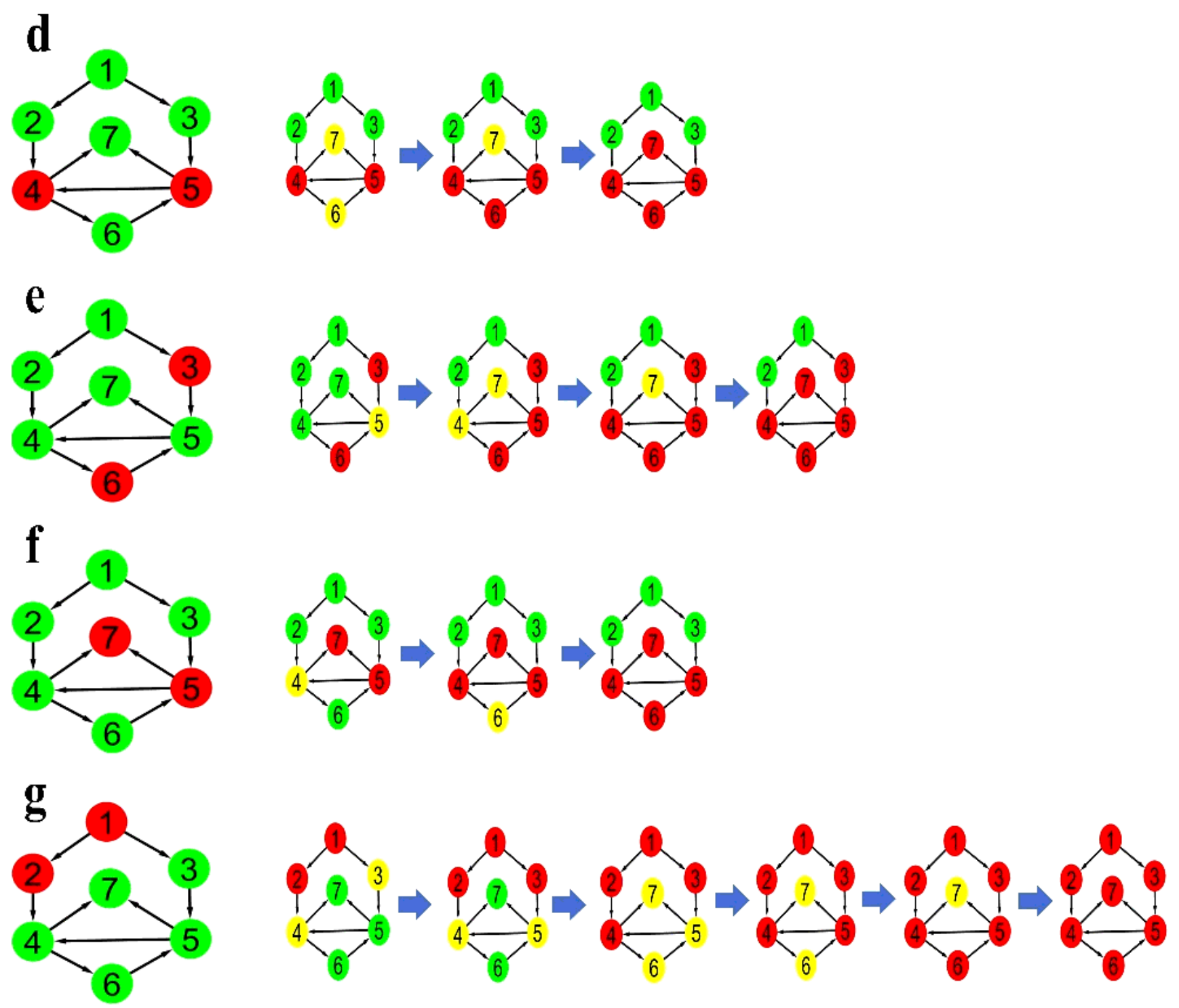

Figure 1

please see the manuscript file for the full caption 
(a)

(c)
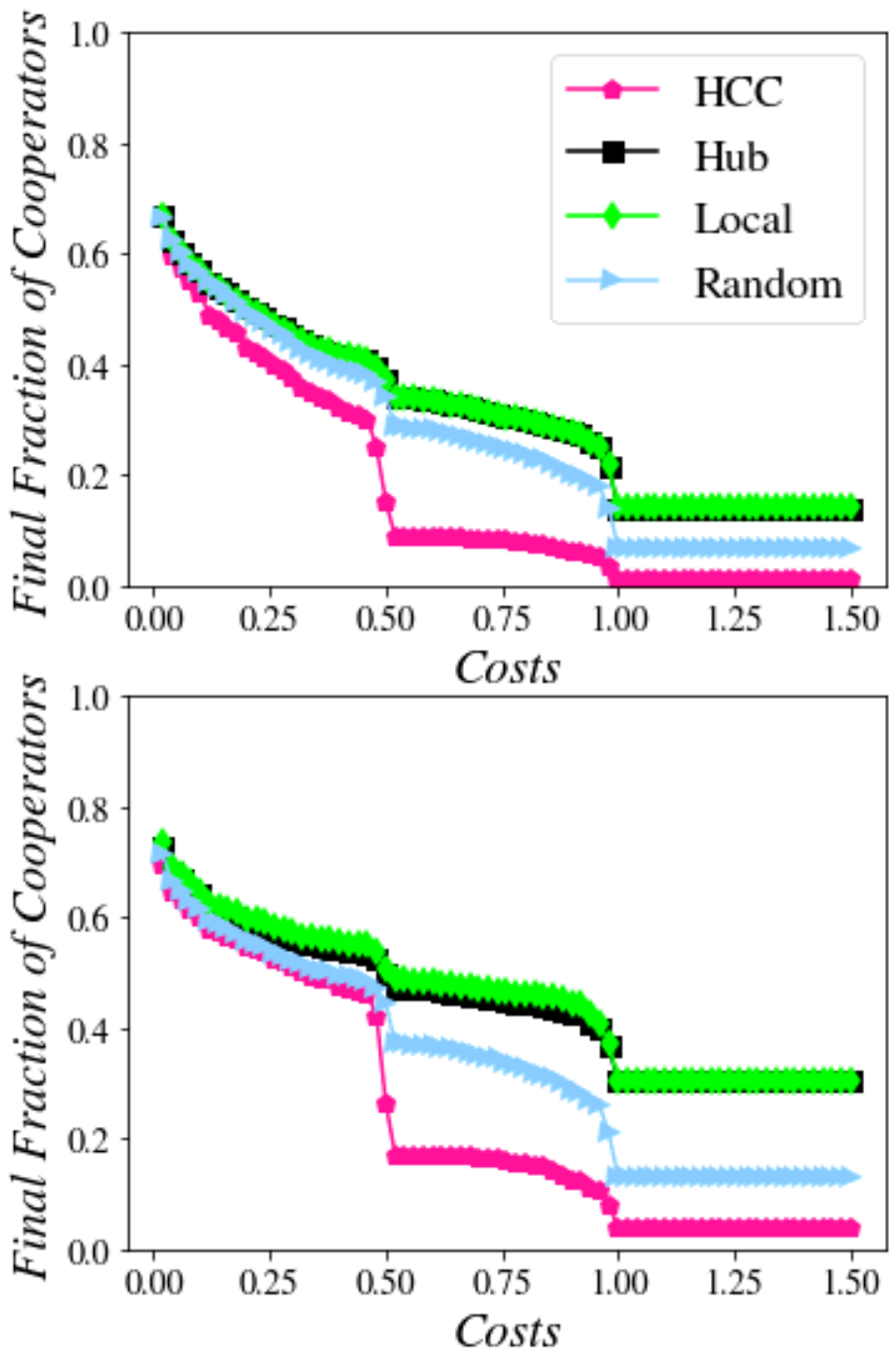

(b)

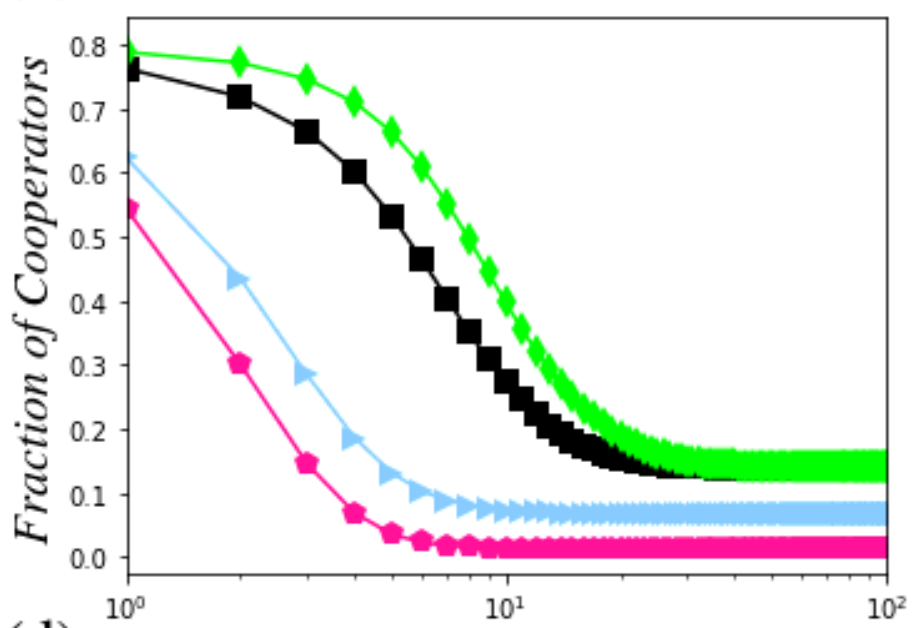

(d)

Time

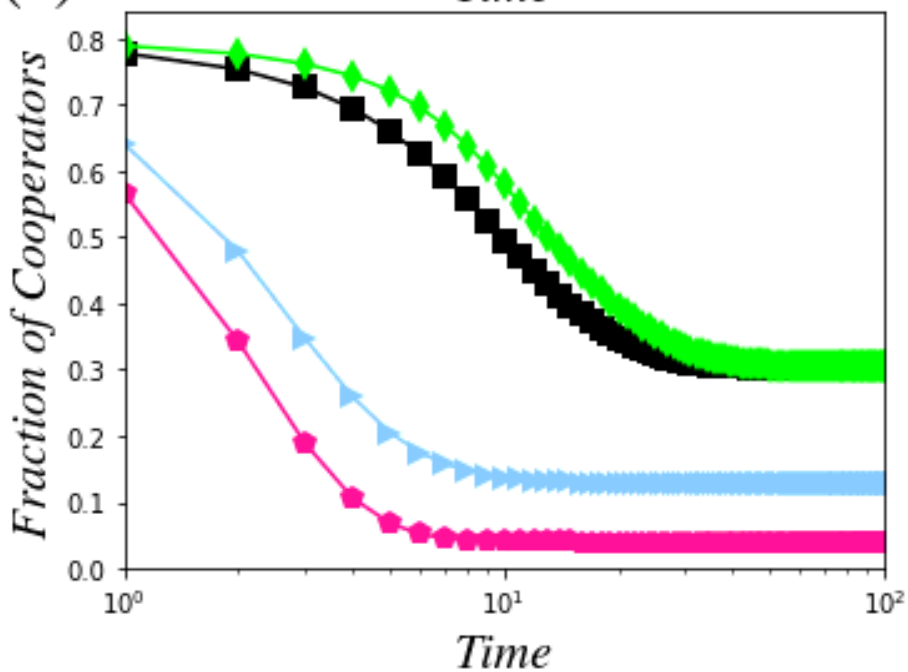

Figure 2

Investigation the effect of initial choice of free-rider nodes in controlling the scale-free networks through deterministic update, for networks with 400 nodes, in dense and sparse networks. a) Analysis of cooperation level in a dense network: Increase in the cooperation cost will lead to decrease in the amount of cooperation. Nodes with the highest control ability, in similar conditions, lead to a lower level of cooperation. b) Analysis of dense network dynamics: if the driver nodes are selected as free-riders, cooperation will decrease ( $c=1)$. c) Analysis of cooperation level in a sparse network: the random sets with the highest control power, whereas by selecting hub and local nodes, a higher level of cooperation is achieved. d) Analyzing the dynamics of a sparse network: just like a dense network, the velocity of achieving a steady state is higher, where nodes with the highest control power are selected $(c=1)$. 
(a)

(c)
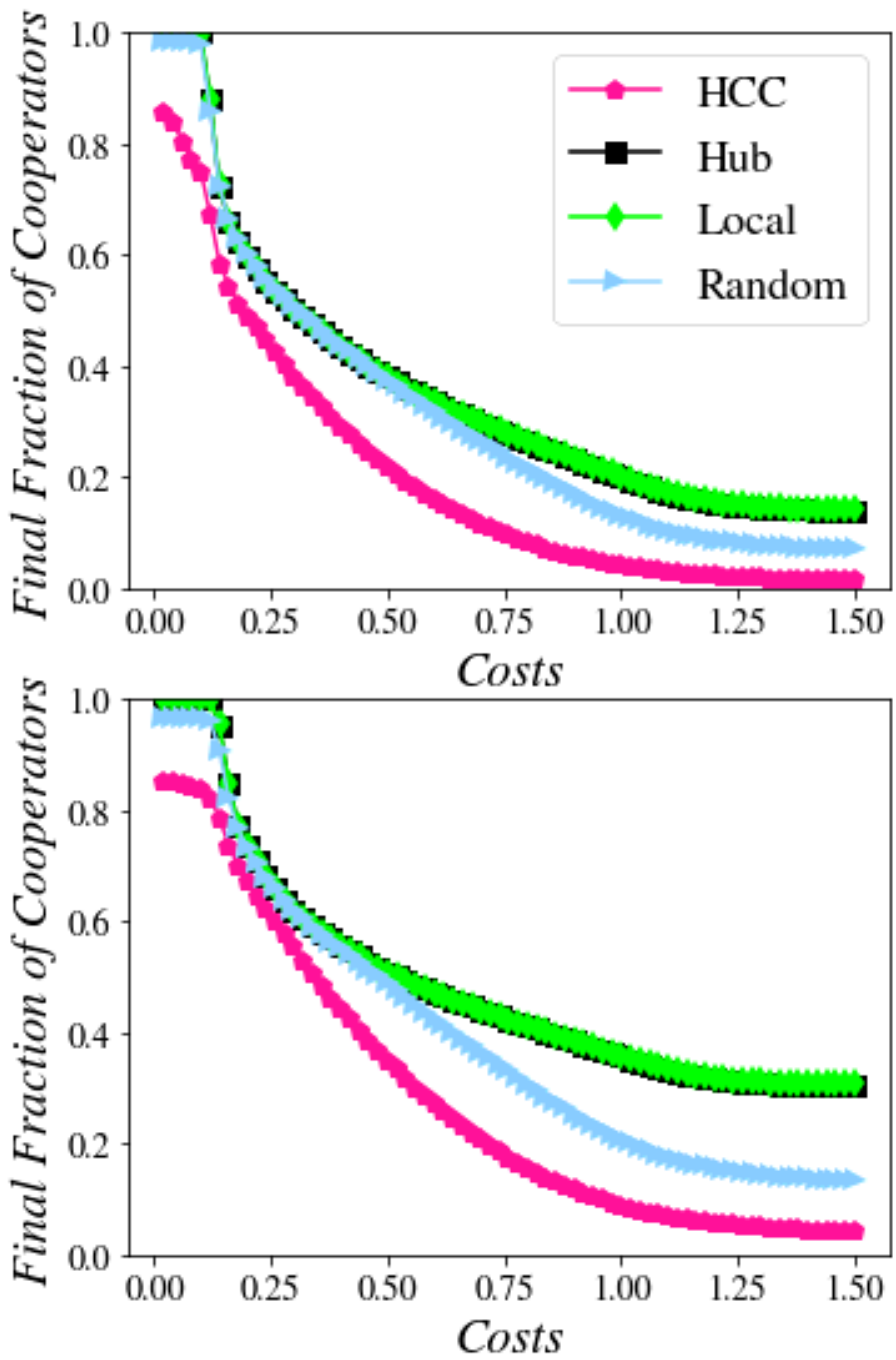

(b)
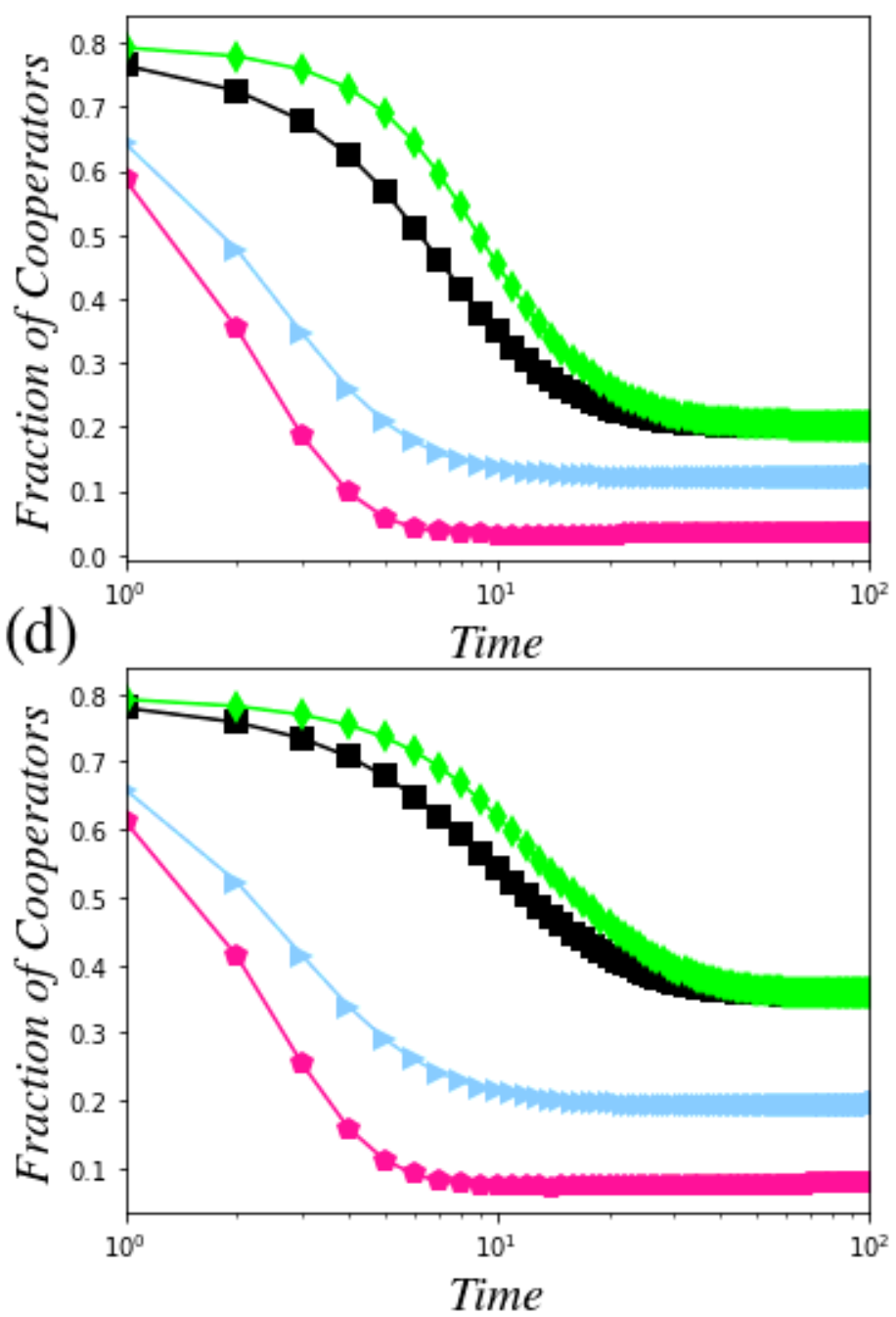

Figure 3

Analyzing the effect of initial node selection in network control using the Glauber update rule. Other conditions are as the ones shown in Fig 2. 
(a)

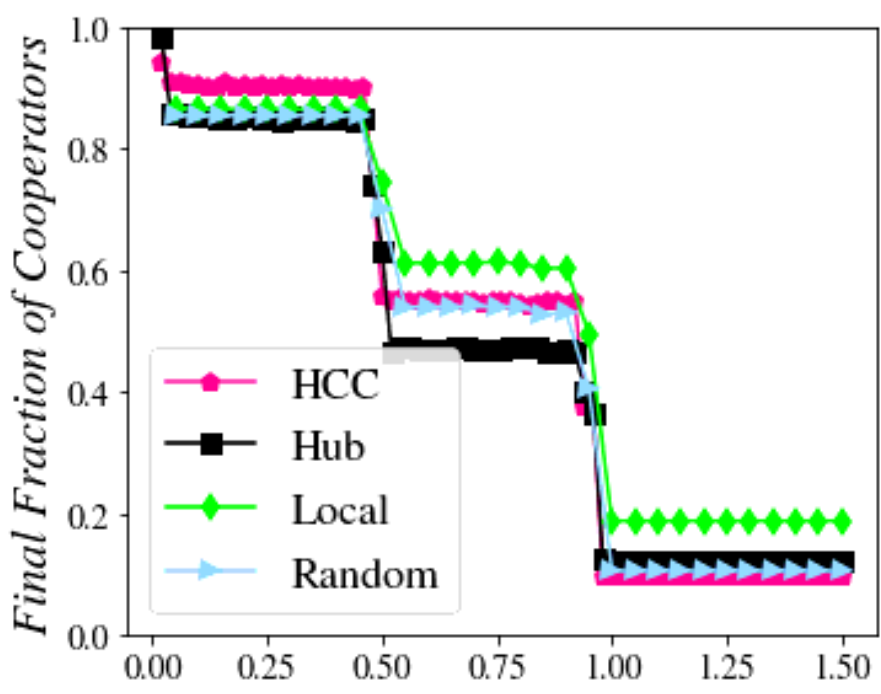

(c)

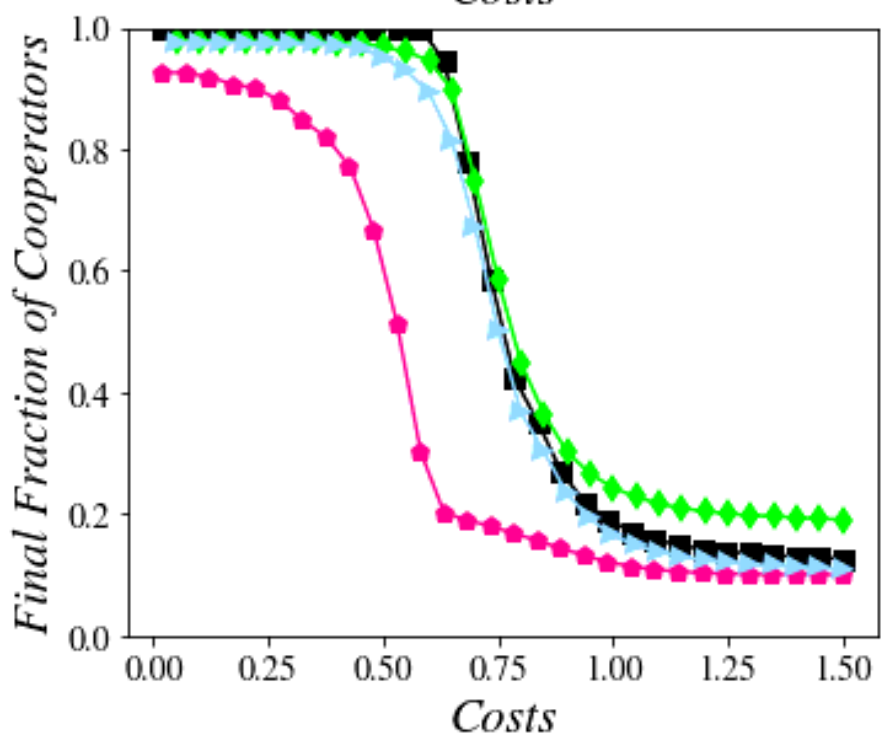

(b)
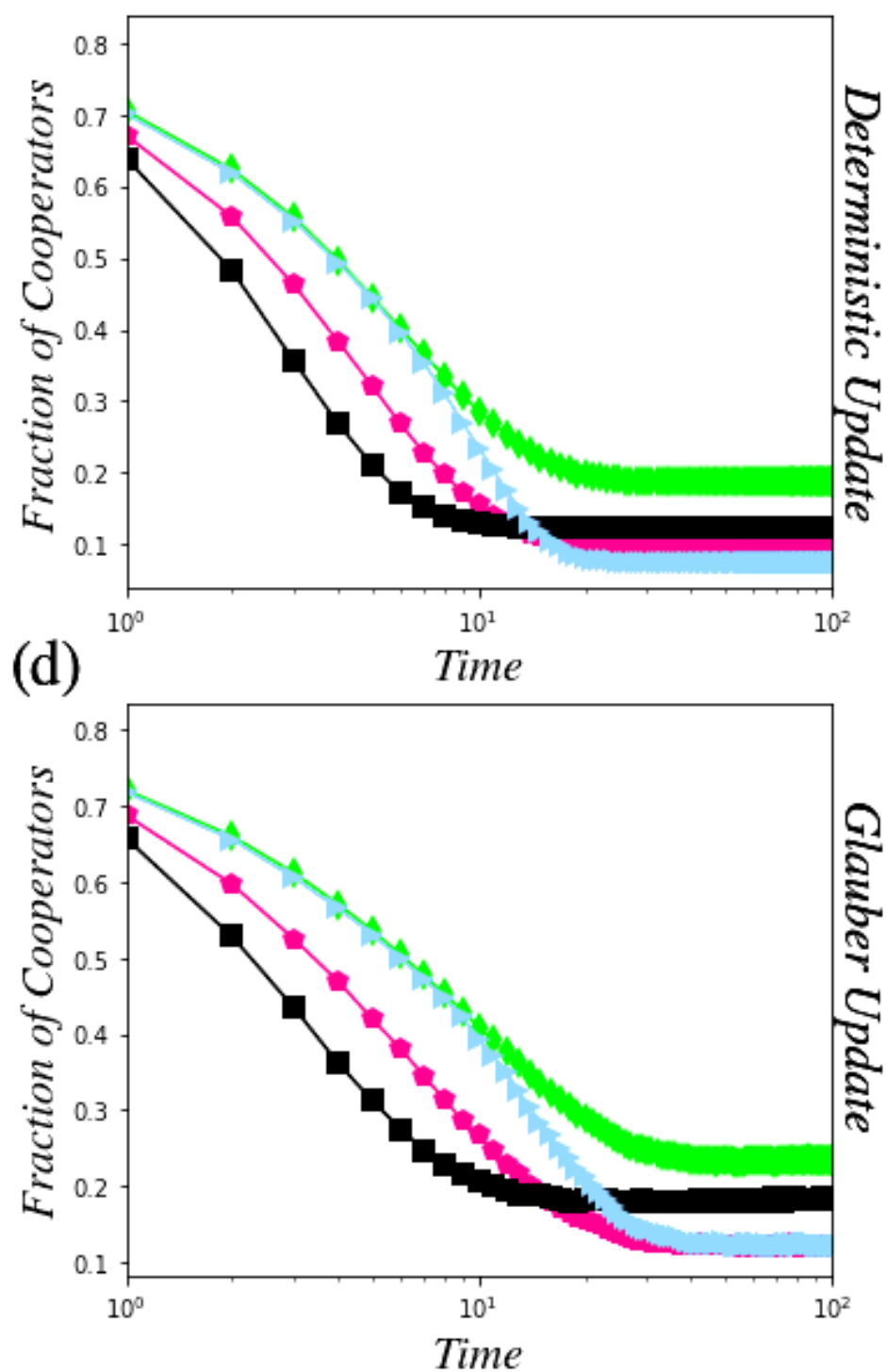

Figure 4

Results regarding to the 4420 network a) Analysis of cooperation level based on the deterministic update rule. b) Network dynamics in achieving steady state relevant to the deterministic update law $(c=1)$. c) Analysis of cooperation level based on the Glauber update rule. d) Network dynamics in achieving steady state related to the Glauber update rule $(c=1)$.

\section{Supplementary Files}

This is a list of supplementary files associated with this preprint. Click to download.

- Supplementary.docx 